Cuadernos de Trabajo Social

ISSN: 0214-0314

\title{
Dificultades de los profesionales de protección infantil en la investigación de casos
}

\author{
Eduardo Martín Cabrera ${ }^{1}$; Haridian Suárez Martín ${ }^{2}$
}

Recibido: 24/05/2016/ Revisado: 06/06/2016/Aceptado: 02/02/2017

Resumen. La investigación de las notificaciones de posibles situaciones de desprotección infantil es una tarea compleja, que es responsabilidad de los profesionales del sistema de protección infantil y que no siempre se puede realizar en las mejores condiciones. El objetivo de este estudio es conocer cuáles son las principales difícultades con las que se enfrentan los profesionales. Para ello, 90 técnicos respondieron a un cuestionario que contenía una pregunta abierta sobre este aspecto. A través del análisis de contenido de las respuestas, se establecieron ocho categorías. El problema más frecuente es el del poco tiempo del que disponen para realizar la investigación, aunque también se indican otras categorías referidas a las condiciones de trabajo y a la falta de formación técnica. Asimismo se encuentran algunas diferencias referentes al perfil profesional. Se discuten los resultados hallados en relación a la literatura previa y se desprenden algunas reflexiones de cara a la mejora de las tareas de investigación

Palabras clave: Maltrato infantil; protección infantil; equipos municipales de protección infantil; investigación; dificultades

\section{[en] Difficulties of professionals of the child welfare system to investigate cases}

\begin{abstract}
Investigating notificatio s of possible situations of child abuse is a complex task for which professionals in the child protection system are responsible. Investigations cannot always be conducted under the best conditions. The aim of this study is to identify the main difficulties that professionals face. For this purpose, 90 specialists responded to a questionnaire consisting of an open question regarding this issue. Eight categories were established pursuant to an analysis of the content of the responses. The most common problem is a lack of available time to conduct investigations, though other categories are identified relating to working conditions and lack of technical training. Some differences are also found with regard to professional profile. The results obtained are discussed with reference to previous literature and reflections are provided in terms of improving investigations
\end{abstract}

Key words: child abuse; child protection; municipal child protection teams; investigation; difficulties

Sumario: Introducción. 1. Método. 1.1. Participantes. 1.2. Instrumento. 1.3. Procedimiento. 2. Resultados. 3. Discusión y conclusiones. 4. Referencias bibliográficas

Cómo citar: Martín Cabrera, E.; Suárez Martín, H. (2018) La investigación en desprotección infantil, en Cuad. trab. soc. 31(1), 189-198.

\section{Introducción}

El maltrato infantil es un problema social que afecta a un número elevado de niños y niñas en todo el mundo, con graves consecuencias para su desarrollo, ya que se trata de una población especialmente vulnerable. Según los datos que maneja la Organización Mundial de la Salud, se estima que en Europa la incidencia del maltrato físico se sitúa sobre el 22,9 por ciento, y

\footnotetext{
Universidad de La Laguna, España edmartin@ull.edu.es

2 Universidad de La Laguna, España

haridiann@hotmail.com
} 
la del abuso sexual en el 9,6 por ciento (World Health Organization, 2014). Aunque se debería considerar como un problema de salud pública, lo que repercutiría en una mejora de los recursos que se dedican para la prevención y la intervención (De Paúl, 2012), lo cierto es que en la actualidad parece casi imposible disponer de datos epidemiológicos fiables, debido fundamentalmente a que no existe un sistema de clasificaci n consensuado (Muela, 2008; Muela, López de Arana, Barandiaran, Larrea y Vitoria, 2012); y a que las administraciones utilizan diferentes criterios para identificar los distintos tipos de maltrato infantil (Fallon Trocmé, Fluke, Maclaurin, Tonmyr y Yuan, 2010). Algo similar ocurre en el caso de España, ya que es muy complejo disponer de estadísticas oficiales completas a nivel nacional (Observatorio de la Infancia, 2011). Ello se debe a la dificultad para recopilar los datos de las diferentes comunidades autónomas mediante fuentes análogas, y utilizando los mismos procedimientos. Aún así, los datos existentes nos permiten hacernos una idea bastante aproximada tanto de la magnitud del problema (Saldaña, Jiménez y Oliva, 1995), como de su compleja realidad (Centro Reina Sofía, 2011) y de las graves consecuencias que tiene en el desarrollo de las víctimas, incluso a largo plazo (; Lázaro y López, 2010; Fresno-Rodríguez, Spencer-Contreras y Retamal-Castro, 2012; Muela, Torres y Balluerka, 2012).

Todo ello resalta la importancia de la labor que desarrollan los profesionales del sistema de protección infantil. En España, los cambios legislativos que se producen a finales de la década de 1980, que promueven la desjudicialización y la descentralización del sistema de protección infantil, otorgan a las entidades locales - los ayuntamientos - la competencia para investigar las sospechas y notificaciones de posibles casos de desprotección infantil.

Las situaciones de desprotección infantil son aquellas en las que hay un comportamiento inadecuado de los adultos que ha provocado o que puede provocar un daño significativo en el niño (Arruabarrena, 2011). Esto convierte a los profesionales municipales en el primer fi tro que decide si un niño o una niña entra en el sistema de protección o no, encargándose también de intervenir en aquellos casos confi mados y menos graves, declarados como casos de riesgo con los programas de intervención familiar, y derivando los casos más graves, para su declaración de desamparo por una Administración de rango superior.

La importancia de su labor se refleja en la gran cantidad de literatura científica sobre los criterios utilizados en la toma de decisiones. A nivel internacional, diversos estudios han comprobado que existen sesgos que pueden provocar errores en la toma de decisiones (Munro, 1999), y que se realizan juicios preconcebidos (Jent, Merrick, Dandes, Lambert, Haney y Cano, 2009). Se ha comprobado también que algunos profesionales tienen mayor tendencia que otros a proponer medidas de separación familiar (Arad-Davidzon y Benbenishty, 2008) encontrándose incluso que, a la hora de clasificar las diferentes formas de desprotección, había desacuerdos entre investigadores y profesionales (Runyan et al., 2005). En nuestro país, Arruabarrena y De Paúl (2011) utilizaron viñetas que representaban diversas situaciones de desprotección infantil, y encontraron que existen diferencias entre los profesionales en valorar la gravedad de las distintas situaciones, no encontrando porcentajes de acuerdo superiores al 80 por ciento en ninguna de las viñetas. Por su parte, Rodríguez, Camacho, Rodrigo, Martín y Máiquez (2006) comprobaron que los técnicos municipales tienden a sobrestimar los factores de riesgo en detrimento de los factores protectores cuando evalúan el riesgo psicosocial.

Otro aspecto, fundamental que dificulta la labor de los profesionales, a la hora de investigar las notificaciones de posibles situaciones de desprotección infantil y tomar decisiones, se refieren a la coordinación horizontal; es decir: entre los servicios sociales de base y los especializados (De Paúl, 2009); o incluso entre los profesionales de los propios equipos que están formados por diferentes perfiles profesionales (Martín y Aciego de Mendoza, 2011). También existen dificultade en la coordinación con otros servicios, ya que son conocidos los problemas que se detectan cuando se busca recabar información de otros profesionales, como pueden ser los del ámbito educativo, sanitario, judicial, policial o de salud mental. Esta coordinación resulta compleja en muchas ocasiones (De Paúl, 2009), siendo necesario establecer mecanismos de entendimiento que faciliten una recogida de información eficaz sobre los casos notificados (Jent et al., 2009; Álvarez, Donohue, Carpenter, Romero, Allen y Coss, 2010; Lalayants, Epstein y Adamy, 2011). 
Todo lo señalado indica que los profesionales encargados de la investigación tienen una enorme responsabilidad y, en muchas ocasiones, trabajan bajo una enorme presión por la cantidad de casos a investigar, la urgencia con la que deben trabajar y, frecuentemente, compatibilizando la investigación con otras tareas que tienen que desarrollar, viéndose con una gran sobrecarga de trabajo (Casas y Montserrat, 2002) y estando dentro de los colectivos profesionales sometidos a una enorme presión y riesgo de sufrir burnout (Lee, Weaver y Hrostowski, 2011).

Una de las demandas de estos profesionales es la de un mayor apoyo y asesoramiento técnico (Anguera, Blanco, Losada, Montilla y De Armas, 1995; Casas y Montserrat, 2002; Martín y Aciego de Mendoza, 2011). En este sentido, se han ido publicando guías y protocolos de evaluación de casos por todo el territorio nacional prácticamente. La investigación ha demostrado que disponer de instrumentos de evaluación eficaces y un buen entrenamiento en el uso de los mismos mejora la valoración de casos y la toma de decisiones (Arruabarrena y De Paúl, 2012; De Kwaadsteniet, Bartelink, Witteman, Ten Berge y Van Yperen, 2013), sobre todo si la implementación de dichos instrumentos se hace partiendo de los contextos en los que se van a aplicar (Gillingham, 2011; Martín y Aciego de Mendoza, 2011).

La Organización Mundial de la Salud, en su Plan de Acción europeo para la prevención del maltrato infantil 2015-2020, recomienda mejorar la formación de los profesionales en la detección y atención temprana al maltrato infantil (World Health Organization, 2014). En esta misma línea, De Paúl (2009) ya planteaba, en un artículo en el que revisaba la evolución y la situación actual del sistema de protección infantil en España, que se debe mejorar la capacidad de los profesionales de los servicios sociales de base para llevar a cabo la investigación y la evaluación de las familias y los menores que se encuentran en una posible situación de desprotección. En este sentido, conocer las dificultades a las que se enfrentan a diario, para poder investigar los casos de posibles situaciones de desprotección, nos permitirá hacer un diagnóstico adecuado sobre qué aspectos se deben mejorar desde el punto de vista de los propios profesionales, siendo este el principal objetivo de este trabajo. Algunos autores señalan que es importante tener en cuenta, además de los instrumentos y los protocolos con los que se trabaja, otras variables referidas a los profesionales. Así, Ogden (2012) resalta que el éxito de cualquier intento de dotar a los profesionales de instrumentos de trabajo depende no solamente de la evidencia empírica que avala dicho instrumento, sino también que se ha de tener en cuenta el contexto en el que se va a usar, así como aquellas variables que pueden facilitar su uso por los profesionales.

Por último, dado que en la actualidad se tiende cada vez más a contar con equipos formados por diferentes perfiles profesionales, en este trabajo también se analizará si existen diferencias en las dificultades con las que se encuentran, en función de los perfiles profesionales.

Concretamente nos centraremos en las dificultades con las que se encuentran los profesionales para realizar la fase de investigación previa. Esta fase inicial, cuyos objetivos fundamentales son: verificar si existe una situación de desprotección y, en caso de que exista, valorar su gravedad, por si fuera necesario adoptar alguna medida protectora de urgencia, quedebe realizarse de manera rápida, en un plazo de 10 ó 15 días (Arruabarrena, 2009).

\section{Método}

\subsection{Participantes}

La muestra de este estudio la forman 90 técnicos de los servicios sociales municipales, que representan el 71,4 por ciento del total de los profesionales municipales con responsabilidades en materia de protección infantil en la isla de Tenerife. En cuanto al perfil profesional, 23 son psicólogos (25 por ciento), 47 trabajadores sociales (51.1 por ciento ) y 19 educadores (20,7 por ciento).

\subsection{Instrumento}

Dentro de un proyecto más amplio, se elaboró un cuestionario ad hoc diseñado para conocer qué procedimiento usan los profesionales del sistema de protección infantil en la investigación de los casos de desprotección infantil. Se añadió una pregunta abierta en la que se les interrogaba acerca de cuáles eran las dificu tades con las que se encontraban a la hora de realizar preguntas de respuesta abierta. En este artículo se analizan las respuestas a la pregunta 
sobre las dificultades que se encuentran para desarrollar la fase de investigación previa de los casos que son notificados

\subsection{Procedimiento}

El cuestionario se les hizo llegar a todos los profesionales que trabajaban en los diferentes ayuntamientos de la isla de Tenerife, a través de la Administración insular competente en materia de protección infantil. Los cuestionarios los realizados de manera voluntaria, individual y anónima los técnicos municipales, y la tasa de respuesta fue el 71,4 por ciento.

Una vez recogidos todos los cuestionarios, se procedió a la categorización de las respuestas a la preguntas sobre las dificultades que encontraban cuando iban a la hora de realizar la fase de investigación previa. A través de la técnica de análisis de contenido, se procedió a la categorización de las respuestas por un proceso inductivo. Para la elaboración final de las categorías se tuvieron en cuenta los criterios de homogeneidad, utilidad, exclusión mutua y claridad, y concreción, recomendados por Pérez (2001).

Se extrajeron ocho categorías que se describen y ejemplifican a continuación

1. Plazo corto: en esta categoría se incluyen todas las referencias al escaso tiempo del que se dispone, como un problema para realizar la investigación previa. Algunos ejemplos de respuestas asignadas a estas categorías son: En 10 días no da tiempo de hacer la investigación previa (C25: Trabajadora Social); El plazo es demasiado corto (C40: Psicólogo); Falta de tiempo (C44: Educadora).

2. Instrumento complejo: se incluyen todas las respuestas que indican que el procedimiento recogido en el Protocolo de valoración de situaciones de desprotección infantil (PVSDI) es un proceso complejo, costoso y difícil de llevar a cabo. Ejemplos de respuesta: Se debería mejorar el proceso de toma de decisión (C2: Psicóloga); Es demasiada la dedicación necesaria para recoger toda la información (C33: Psicóloga); El tener que plasmar por escrito el procedimiento y la planificació (C30: Trabajador Social).
3. Instrumento incompleto: se hace referencia a que el Protocolo de valoración de situaciones de desprotección infantil carece de algún aspecto importante, como pueden ser variables a estudiar, hojas de registro, instrumentos de recogida de información, etc. Ejemplos de respuestas asignadas a esta categoría son: No se analizan algunas variables educativas en profundidad (C5: Educadora); Es complicado identificar los factores e indicadores (C30): Trabajador Social; No se recoge información sobre las fortalezas (C56: Psicólogo).

4. Trabajo con las familias: en esta categoría se incluyen todas las dificultades debidas a la actitud y el comportamiento de las familias a la hora de investigar la situación notificada. Algunos ejemplos son: Poca colaboración de algunas familias (C5: Educadora); La disposición y colaboración de las familias (C7: Trabajadora social); Inasistencia de las familias a las citaciones (C17: Psicóloga)

5. Falta de hábito o formación: esta categoría aglutina todas las respuestas que hacen referencia a que no se está habituado a trabajar con el instrumento, que no se han familiarizado con el Protocolo de valoración de situaciones de desprotección infantil y/o que se requiere formación. Algunos ejemplos de respuestas son: Falta de tiempo para asimilar los conceptos teóricos (C18: Educadora); Esto supone un cambio en el sistema de trabajo habitual (C41: Trabajadora Social); Poca familiarización con el instrumento (C44: Educador).

6. Coordinación interna: aquí se incluyeron todas las respuestas que hacían referencia a las dificultades para trabajar como equipo interdisciplinar, debido a aspectos que van desde la falta de algún perfil, hasta la falta de entendimiento o de tiempo y espacios para reunirse. Algunos ejemplos son: Hacer coincidir las agendas de los miembros del equipo (C47: Psicóloga); El trabajo conjunto de las tres disciplinas (C127: Trabajadora Social); Falta la figura del psicólogo en el equipo (C102: Trabajador Social).

7. Coordinación externa: esta categoría incluye todas las respuestas referidas a las difi- 
cultades para recabar información y trabajar de manera coordinada con otros recursos externos al ámbito municipal. Ejemplos de estas respuestas son: Hay poca implicación de algunas fuentes y recursos con los que se contacta para pedir información (C4: Trabajadora Social); La recogida de información depende de la disponibilidad de los diferentes recursos (C22: Trabajador Social); Encontrar canales de comunicación con determinados recursos (C69: Educadora).

8. Condiciones de trabajo: en esta última categoría se agrupan todas las respuestas referidas a las condiciones laborales como pueden ser la sobrecarga de trabajo, la falta de personal, las condiciones materiales y de espacio, etc. Ejemplos: Es dificil compaginar una investigación previa en la que hay que llevar un registro tan minucioso con el trabajo del día a día (C24: Trabajadora Social); Hay un excesivo número de expedientes abiertos (C34: Educadora); Es imposible llevar a cabo todo el proceso de investigación previa con todos los casos porque somos solo tres profesionales en el equipo (C35; Trabajador Social).

Una vez establecidas las categorías, dos jueces ciegos asignaron las respuestas a las mismas. Para analizar la fiabilidad del procedimiento se procedió a hallar el índice Kappa, encontrando valores adecuados en todas las categorías (véase Tabla 1) ya que varían entre sustanciales y casi perfectos según la clasificación de Landis y Koch (1977). Posteriormente cada categoría se convirtió en una variable dicotómica, a la que se le asignó un valor de 1 si la respuesta del profesional pertenecía a dicha categoría y de 0 si no pertenecía.

Tabla 1. Grado de acuerdo entre jueces

\begin{tabular}{ll}
\hline Categorías & Índice Kappa \\
\hline Plazo corto & 0,973 \\
Instrumento complejo & 0,782 \\
Instrumento incompleto & 0,752 \\
Trabajo con las familias & 0,851 \\
Falta de hábito/formación & 0,752 \\
Coordinación interna & 0,722 \\
Coordinación externa & 0,840 \\
Condiciones de trabajo & 0,774 \\
\hline
\end{tabular}

\section{Resultados}

En el Gráfico 1 se puede observar el porcentaje de la muestra que alude a cada una de las categorías. Se puede comprobar que la gran mayoría alude a la categoría "plazo corto". La segunda categoría nombrada con más frecuencia es "instrumento complejo", seguida de "condiciones de trabajo" y "falta de hábito/ formación", categorías a las que alude aproximadamente un tercio de la muestra. Les siguen las categorías de "instrumento incompleto", "coordinación interna" y "coordinación externa", que las señala aproximadamente uno de cada cinco encuestados. La categoría a las que menos alusiones hacen, con diferencia, es la que se refiere a "trabajo con las familias".

Con el objetivo de comprobar si las dificultades que encuentran para realizar la investigación previa varían en función del perfil profesional, se realizaron contrastes $J i$ ferencias estadísticamente significativas en tres categorías. La categoría "plazo corto", que la señalan menos los educadores que los psicólogos y los trabajadores sociales $\left(\chi_{(2)}^{2}=6.427 ; p<.05\right)$, la categoría "coordinación interna” que la señalan más los psicólogos y los educadores que los trabajadores sociales $\left(\chi_{(2)}^{2}=8.008 ; p<.05\right)$, y a la categoría "coordinación externa", aluden menos los psicólogos que los educadores y los trabajadores sociales: $\chi_{(2)}^{2}=1.508 ; p<.05$. Para el resto de categorías no se encuentran diferencias significativas: "instrumento complejo": $\chi_{(2)}^{2}=3.199 ; p>.05$; "instrumento incompleto": $\chi_{(2)}^{2}=1.656 ; p>.05$ : "trabajo con las familias": $\chi_{(2)}^{2}=1.585 ; p>.05$; "falta de hábito/formación": $\chi_{(2)}^{2}=1.164 ; p>.05 ; y$ "condiciones de trabajo": $\chi_{(2)}^{2}=3.199 ; p>.05$. 


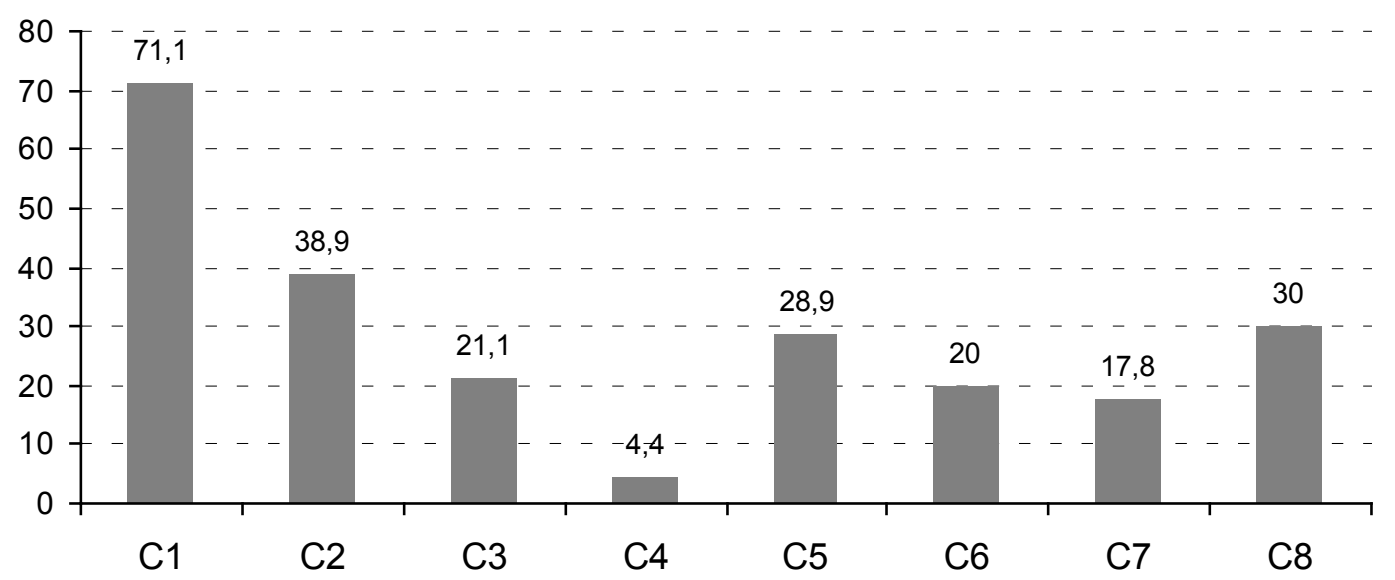

Gráfico 1. Porcentaje de la muestra que alude a los diferentes problemas en la fase de investigación previa

Nota: C1: Plazo corto; C2: Instrumento complejo; C3: Instrumento incompleto; C4: Trabajo con las familias; C5: Falta de hábito/formación; C6: Coordinación interna; C7: Coordinación externa; C8: Condiciones de trabajo

Tabla 2. Diferencias entre los perfiles profesionales en las categorías de problema

\begin{tabular}{|c|c|c|c|}
\hline & \multicolumn{3}{|c|}{ Porcentaje de cada perfil que alude a cada categorí } \\
\hline & Psicólogo & Educador & Trabajador Social \\
\hline Plazo corto & 78,3 & 47,4 & 76,6 \\
\hline Instrumento complejo & 30,4 & 26,3 & 46,8 \\
\hline Instrumento incompleto & 30,4 & 21,1 & 17 \\
\hline Trabajo con las familias & 8,7 & 5,3 & 2,1 \\
\hline Falta de hábito/formación & 21,7 & 36,8 & 29,8 \\
\hline Coordinación interna & 26,1 & 36,8 & 8,5 \\
\hline Coordinación externa & 8,7 & 21,1 & 19,1 \\
\hline Condiciones de trabajo & 26,1 & 36,8 & 29,8 \\
\hline
\end{tabular}

\section{Discusión y conclusiones}

El primer objetivo de este trabajo era conocer las dificultade con las que se encuentran los profesionales del sistema de protección infantil a la hora de investigar las notificaciones de posibles situaciones de desprotección infantil. Los resultados de este trabajo muestran que los problemas con los que se encuentran los téc- nicos municipales son variados y se deben a diferentes factores.

En primer lugar, habría que decir que el problema más habitual, con diferencia, es el que se refiere a los plazos que se estipulan habitualmente para realizar la primera fase de la investigación previa, que suelen situarse entre 10 y 15 días (Arruabarrena, 2009). Lógicamente esto no se debe cambiar, ya que el ob- 
jetivo es que no pase demasiado tiempo, desde que llega la notificación hasta que se termina esta primera fase, para poder dar una respuesta lo más inmediata posible a aquellos casos realmente graves, sobre todo los que requieren una intervención inmediata, como puede ser la separación del menor de su familia, debido a que corre riesgo de sufrir un daño grave, o cuando se comprueba que ya lo está sufriendo. Para el resto de casos en los que se observa que existe desprotección, pero sin riesgo de daño importante, se comienza una segunda fase de valoración con mayor profundidad y de plazos más largos. Dado que aún así los profesionales aluden al corto tiempo del que disponen para realizar la fase de investigación previa, parece conveniente analizar este problema en relación con otros, sobre todo con los más frecuentes. Así, dos de los problemas más nombrados hacen referencia, por un lado, a lo complejo que les resulta el instrumento que tienen que usar; $\mathrm{y}$, por el otro, a la falta de hábito para trabajar con el mismo y a la falta de formación. Estos problemas están claramente relacionados con la demanda que plantean los profesionales del sistema de protección infantil de obtener un mayor apoyo y asesoramiento técnico para realizar una labor tan compleja como la que afrontan y que tienen que finalizar en un corto espacio de tiempo (Anguera, Blanco, Losada, Montilla y De Armas, 1995; Casas y Montserrat, 2002; De Paúl, 2009; Martín y Aciego de Mendoza, 2011). Este parece ser un aspecto todavía no resuelto completamente, aunque se ha demostrado que la formación y el asesoramiento mejoran la eficacia en las tareas de investigación y de toma de decisiones (Arruabarrena y De Paúl, 2012; De Kwaadsteniet, Bartelink, Witteman, Ten Berge y Van Yperen, 2013), sobre todo cuando se realiza de una manera contextualizada, partiendo de la realidad cotidiana con la que se enfrentan los profesionales del sistema de protección infantil (Hernández, 1993; Gillingham, 2011; Martín y Aciego de Mendoza, 2011; Ogden, 2012).

Otro problema al que alude cerca de un tercio de los profesionales es el de las condiciones de trabajo. La sobrecarga de tareas y la falta de recursos también les dificultan realizar la investigación previa en condiciones adecuadas. Este problema es ampliamente reconocido y les hace trabajar bajo una enorme presión, sobre todo si se tiene en cuenta la importancia de la labor que desarrollan (Casas y Montserrat, 2002; Lee, Weaver y Hrostowski, 2011).
Lo realmente preocupante es que, con la actual crisis económica, la situación no solo no mejore sino que probablemente empeore, debido a los recortes que sufren ya los servicios sociales municipales.

Por otra parte, aproximadamente uno de cada cinco profesionales alude a los problemas de coordinación, interna y externa, como un factor que dificulta su labor de investigación. Con respecto a la coordinación interna, parece que un número considerable de profesionales sigue manifestando problemas para coordinarse con sus compañeros de equipo, bien debido a la falta de cultura de trabajo en equipo, bien por las diferentes formas de trabajo que se establecen en las diversas disciplinas (Martín y Aciego de Mendoza, 2011). Incluso los problemas que se indican en la categoría de condiciones de trabajo pueden estar detrás de esto, sobre todo los que aluden a la sobrecarga de trabajo que dificult incluso buscar espacios, tanto físicos como temporales, para poder reunirse. En lo que se refiere a los problemas de coordinación externa, los resultados de este trabajo no son una novedad, ya que es un problema ampliamente reconocido (De Paúl, 2009; Jent, et al., 2009; Álvarez et al., 2010; Lalayants, Epstein y Adamy, 2011; World Health Organization, 2014).

La categoría menos aludida es la referida al trabajo con las familias, hecho que debe interpretarse de manera positiva. Aunque este aspecto ha sido tradicionalmente considerado un problema para estos profesionales, debido a los recelos y la desconfianza que manifie tan las familias hacia los profesionales (Arruabarrena, 2011), el conocer el porqué de estas reacciones y el desarrollar metodologías de trabajo cooperativo con las familias, con las que se comienza a trabajar con un enfoque positivo, parece estar dando buenos resultados (Rodrigo, Máiquez, Martín y Byrne, 2008) para lograr la colaboración de las familias en los procesos de investigación.

El segundo de los objetivos planteados en este trabajo era comprobar si existen diferencias en los problemas que se encentran para realizar la investigación previa en función del perfil profesional. Los resultados confirman que dichas diferencias existen, concretamente en tres de las categorías. Así, los educadores señalan en menor medida el plazo establecido como un problema, en comparación con los psicólogos y los trabajadores sociales. Una 
explicación para este hecho puede ser que la investigación que realizan tanto los psicólogos como los trabajadores sociales es más compleja y requiere una recogida de información más amplia que la que realiza el educador, que en muchos casos se centra exclusivamente en la situación escolar del menor.

Otra categoría en la que se observan diferencias significativas es la de coordinación interna, a la que los trabajadores sociales aluden menos que los psicólogos y educadores. Esto se debe probablemente a que el perfil competencial y de tareas del trabajador social está claro, mientras que pueden existir problemas de definición profesional del educador, que en muchas ocasiones valora e investiga variables tradicionalmente asignadas al psicólogo. Hay que decir aquí que en el contexto en el que se realizó este trabajo, Tenerife, no existe la titulación de educador social, y que a las plazas de educador pueden acceder varios perfiles profesionales, como psicólogos, pedagogos, maestros, etc.

La última diferencia encontrada se da en la categoría coordinación externa, que es menos señalada por los psicólogos. Parece claro que son los trabajadores sociales los que tienen que coordinarse con los servicios de sanidad y los educadores con los educativos, refiriendo muchos de ellos que dichos servicios tardan en remitir la información más tiempo del estipulado para realizar la investigación previa. Por su lado, los psicólogos se encargan de coordinarse con los servicios de salud mental, hecho que no es necesario en la mayoría de los casos estudiados.

Dos son las principales conclusiones que se desprenden de este trabajo. En primer lugar, se ha constatado que las tareas de investigación previa que realizan los profesionales del sistema de protección infantil se ven dificultadas por múltiples aspectos. Algunos de ellos se refieren a la falta de recursos, tanto personales como materiales, que generan una sobrecarga de trabajo en los profesionales. Este aspecto es especialmente preocupante, pues la crisis económica está afectando especialmente a las administraciones municipales, que son las responsables de realizar la investigación de las notificaciones de posibles situaciones de desprotección infantil.

Otro grupo de problemas tienen un carácter más técnico. Así, parece necesaria una mejora en la formación, tanto en los instrumentos que se usan como en la cultura de trabajo en equipo; tanto de manera interna, donde diferentes perfiles profesionales trabajan para realizar una misma tarea, como concienciando y facilitando la colaboración de otros servicios externos, buscando una participación multisectorial en el abordaje del maltrato infantil (World Health Organization, 2014).

La segunda conclusión hace referencia a la configuraci $n$ de equipos multidisciplinares de protección infantil. Aunque parece positivo debido a la complejidad de los casos investigados, este trabajo demuestra que habría que clarificar las funciones de algún perfil, principalmente las del educador, para impedir problemas de coordinación con otros perfiles, sobre todo con los psicólogos. En una situación tan sensible como es el hecho de entrar en la intimidad de las familias para realizar la investigación, hay que evitar situaciones como las de que dos profesionales recojan la misma información en dos visitas diferentes, hecho que desgraciadamente se da con más frecuencia de lo deseable.

No queremos finalizar sin referirnos a las principales limitaciones de este trabajo. En primer lugar, este estudio se realiza en una comunidad autónoma concreta. El desarrollo de los sistemas de protección infantil ha tenido un desarrollo similar en todas las comunidades autónomas, aunque no igual, por lo que sería deseable replicar esta investigación en otros contextos. En segundo lugar, sería deseable investigar con mayor profundidad y con otras metodologías, como pueden ser los grupos de discusión o las entrevistas en profundidad, para conocer mejor la problemática con la que se enfrentan los profesionales municipales de protección infantil. Por último, se podría analizar también el papel que desempeñan en la percepción de dificultades algunas variables como pueden ser la experiencia, la ratio profesional-casos o la exclusividad en la dedicación a la protección infantil. 


\section{Referencias bibliográfica}

Álvarez, K.M., Donohue, B., Carpenter, A., Romero, V., Allen, D.N. y Coss, C. (2010). Development and preliminary evaluation of a training method to assist professionals in reporting suspected child maltreatment. Child Maltreatment, 15(3), 211-218. doi:10.1177/1077559510365535

Anguera, M.T., Blanco, A., Losada, J.L., Montilla, M.D. y De Armas, M. (1995). Los profesionales del sistema de atención social a la infancia. Realidades y demandas. Madrid: Ministerio de Asuntos Sociales.

Arad-Davidzon, B. y Benbenishty, R. (2008). The role of workers' attitudes and parent and child wishes in child protection workers' assessment and recommendation regarding removal and reunification. Children and Youth Services Review, 30, 107-121. doi:10.1016/j.childyouth.2007.07.003

Arruabarrena, M.I. (2009). Procedimiento y criterios para la evaluación y la intervención con familias y menores en el ámbito de la protección infantil. Papeles del Psicólogo, 30, 13-23.

Arruabarrena, M. I. (2011). Maltrato psicológico a los niños, niñas y adolescentes en la familia: definición y valoración de su gravedad. Psychosocial Intervention, 20(1), 25-44. doi:10.5096/in2011v20n1a3

Arruabarrena, M.I. y De Paúl, J. (2011). Valoración de la gravedad de las situaciones de desprotección infantil por los profesionales de los Servicios de Protección Infantil. Psicothema, 23(4), 642-647.

Arruabarrena, M.I. y De Paúl, J. (2012). Improving accuracy and consistency in child maltreatment severity assessment in child protection services in Spain: New set of criteria to help caseworkers in substantion decisions. Children and Youth Services Review, 34, 666-674. doi:10.1016/j.childyout.2011.12.011

Casas, F. y Montserrat, C. (2002). Interdisciplinary teams and decision making in child protection systems: The experience of Catalonia. International Journal of Child and Family Welfare, 5, 146-158.

Centro Reina Sofia. (2011). Maltrato infantil en la familia en España. Madrid: Ministerio de Sanidad, Política Social e Igualdad.

De Kwaadsteniet, L., Bartelink, C., Witteman, C., Ten Berge, I. y Van Yperen, T. (2013). Improved decision making about suspected child maltreatment: results of structuring the decision process. Children and Youth Services Review, 35, 347-352. doi:10.1016/j.childyouth.2012.11.015

De Paúl, J. (2012). Calidad y eficiencia en los programas de atención a la infancia. Ponencia presentada al XI Congreso Internacional de Infancia Maltratada. Oviedo, 17-19 de octubre.

De Paúl, J. (2009). La intervención psicosocial en protección infantil en España: Evolución y perspectivas. Papeles del Psicólogo, 30, 4-12.

Fallon, B., Trocmé, N., Fluke, J., Maclaurin, B., Tonmyr, L. y Yuan, Y. (2010). Methodological challenges in measuring child maltreatment. Child Abuse and Neglect, 34, 70-79. doi:10.1016/j.chiabu.2009.08.008

Freno-Rodríguez, A., Spencer-Contreras, R. y Retamal-Castro, T. (2012). Maltrato infantil y representaciones de apego: defensas, memoria y estrategias, una revisión. Universitas Psychologica, 11(3), 829-838.

Gillingham, P. (2011). Decision-Making tools and the development of expertise in child protection practitioners: are we "just breeding workers who are good at ticking boxes"? Child and Family Social Work, 16(4), 412-421. doi:10.1111/j.1365-2206.2011.00756.x

Jent, J.F., Merrick, M.T., Dandes, S.K., Lambert, W.F., Haney, M.L. y Cano, N.M. (2009). Multidisciplinary assessment of child maltreatment: a multi-site pilot descriptive analysis of the Florida Child Protection Team Model. Children and Youth Services Review, 31, 896-902. doi:10.1016/j.childyouth.2009.04.003

Lalayants, M., Epstein, I. y Adamy, D. (2011). Multidisciplinary consultation in child protection: a clinical data-mining evaluation. International Journal of Social Welfare, 20, 156-166. doi:10.1111/j.14682397.2010.00741.x

Landis, J.R. y Koch, G.G. (1977). The measurement of observer agreement for categorical data. Biometrics, 33, 159-174.

Lázaro, S. y López, F. (2010). Continuidad de los efectos del maltrato durante la infancia en adolescentes acogidos en centros de protección. Infancia y Aprendizaje, 33(2), 255-268. doi:10.1174/021037010791114599

Lee, J., Weaver, C. y Hrostowski, S. (2011). Psychological empowerment and child welfare workers outcomes: a path analysis. Child and Youth Care Forum, 40(6), 479-497. doi:10.1007/s10566-011-9145-7

Martín, E. y Aciego de Mendoza, R. (2011). Evaluación de un protocolo para valorar situaciones de desprotección infantil: la opinión de los técnicos. Universitas Psychologica, 10(3), 851-862.

Muela, A. (2008). Hacia un sistema de clasificación nosológico de maltrato infantil. Anales de Psicología, 24(1), 77-87. 
Muela, A., López de Arana, E., Barandarian, A., Larrea, I. y Vitoria, J.R. (2012). Definition, incidence and psychopatological consequences of child abuse and neglect. En: A. Muela (ed.), Child Abuse and Neglect: a multidimensional approach (pp. 1-18). Rijeka: InTech. doi:10.5772/46135

Muela, A., Torres, B. y Balluerka, N. (2012). Estilo de apego y psicopatología en adolescentes víctimas de maltrato infantil. Infancia y Aprendizaje, 35(4), 451-469. doi:10.1174/021037012803495294

Munro, E. (1999). Common errors of reasoning in child protection work. Child Abuse and Neglect, 23, 745758. doi:10.1016/S0145-2134(99)00053-8

Observatorio de la Infancia. (2011). Boletín de datos estadísticos de medidas de protección a la infancia. Madrid: Dirección General de Servicios para la Familia y la Infancia.

Ogden, T. (2012). Applying an implementation perspective to improve services-integrating research, policy and practice. Ponencia presentada a la XI EUSARF International Conference. Glasgow, 4-7 september.

Pérez, G. (2001). Investigación cualitativa. Retos e interrogantes II. Técnicas y análisis de datos. Madrid: La Muralla.

Rodrigo, M.J., Máiquez, M.L., Martín, J.C. y Byrne, S. (2008). Preservación familiar. Un enfoque positivo para la intervención con familias. Madrid: Pirámide.

Rodríguez, G., Camacho, J., Rodrigo, M.J., Martín, J.C. y Máiquez, M.L. (2006). Evaluación del riesgo psicosocial en familias usuarias de los Servicios Sociales Municipales. Psicothema, 18, 200-206.

Runyan, D.K., Cox, C.E., Dubowitz, H., Newton, R.R., Upadhyaya, M., Kotch, J.B., Leeb, R.T., Everson, M.D. y Knight, E.D. (2005). Describing maltreatment: Do child protective service report and research definitions agree? Child Abuse and Neglect, 29, 461-477. doi:10.1016/j.chiabu.2004.06.015

Saldaña, D., Jiménez, J. y Oliva, A. (1995). El maltrato infantil en España: un estudio a través de los expedientes de menores. Infancia y Aprendizaje, 18(3), 59-68. doi.: 10.1174/02103709560575488

World Health Organization (2014). Investing in children: the European child maltreatment prevention action plan 2015-2020. Recuperado de: http://www.pnsd.msssi.gob.es/novedades/pdf/Investing_in_children_EuropeanActionPlan.pdf (Consultado el 27/12/2014) 